\title{
Les Chironomidés (Diptera) de l'Aude, rivière méditerranéenne des Pyrénées orientales : étude biocénotique et typologique
}

\author{
J.-M. Gendron ${ }^{1}$ \\ H. Laville ${ }^{1}$
}

Mots clés : Diptera, Chironomidae, exuvies nymphales, rivière Aude, Pyrénées, biodiversité, biocénoses, typologie.

Les peuplements chironomidiens des cours supérieur et moyen de l'Aude, rivière méditerranéenne des Pyrénées orientales ont été étudiés à l'aide de récoltes de dérives d'exuvies nymphales.

7 collections à 12 stations ( $1700 \mathrm{~m}$ à $80 \mathrm{~m}$ ) ont été effectuées sur une période d'une année (février à décembre 1989).

21189 exuvies ont été triées et 125 espèces ou taxa identifiés. Parmi elles, 14 sont nouvelles pour la France et 13 pour les Pyrénées (Zone $2 \geq 1000 \mathrm{~m}$ Limnofauna Europaea).

Deux classifications ascendantes hiérarchiques ont permis de définir 4 niveaux typologiques et 5 groupes d'espèces parmi les 52 espèces les plus fréquentes $(\geq 1 \%)$. 8 espèces sont euryzonales, 13 sont caractéristiques de la plaine, 9 sont euryzonales de montagne, 3 sont caractéristiques du piémont et 19 sont réparties en haute et moyenne montagne.

Le peu d'espèces caractéristiques (12/52) et la large répartition altitudinale de la majorité d'entre elles suggèrent un modèle de répartition plutôt clinal que zonal. La zonation typologique de la rivière Aude parait être corrélée avec le climat, la géologie et les étages de végétation du bassin versant.

Le caractère méditerranéen de l'Aude, avec ses températures estivales plus élevées, facilite la remontée altitudinale des espèces qui vivent normalement dans des zones inférieures dans les rivières des Pyrénées centrales et atlantiques.

Chironomidae (Diptera) of the Aude, a mediterranean river from eastern Pyrenees : biocoenotical and typological study

Keywords : Diptera, Chironomidae, pupal exuviae, River Aude, Pyrenees, biodiversity, biocoenosis, biotypology.

Seven collections of chironomid pupal exuviae at twelve sites $(1700 \mathrm{~m}$ to $80 \mathrm{~m})$ on the River Aude were carried out over a one year period (February to December 1989). 21189 exuviae were sorted and 125 species identified. Among them fourteen are new for France and thirteen for the Pyrenees (Zone $2 \geq 1000 \mathrm{~m}$ Limnofauna Europaea).

Two cluster analyses allow the recognition of four typological zones and five species groups based on the distribution of the most frequently occurring $(\geq 1 \%)$ species. Eight species are euryzonal, thirteen are characteristic of the plain, nine are mountain euryzonal, three are characteristic of the foothills and nineteen have an upper and mid mountain distribution.

The few characteristic species (12/52) and the large altitudinal distribution of the majority of these suggests a clinal rather than zonal pattern. The typological zonation of the River Aude seems to be correlated with climate, geology and vegetation zones of the drainage basin.

The mediterranean nature of the river, with higher summer temperatures, facilitates the ascent of species normally living in lower zones in the central and atlantic Pyrenees.

\section{Introduction}

L'étude des communautés de Chironomidés de la rivière Aude dans les Pyrénées orientales constitue le troisième volet du triptyque consacré à ce groupe de diptères dans les torrents et rivières du versant nord pyrénéen.

\footnotetext{
1. Centre d'Ecologie des Systèmes Aquatiques Continentaux, UMR C 5576 CNRS - Université Paul Sabatier, 118 route de Narbonne, F - 31062 Toulouse Cedex 04, France.
}

Les deux premièrs volets concernent les systèmes hydrographiques de la vallée d'Aure dans les Pyrénées centrales (Laville \& Lavandier 1977, Gazagnes \& Laville 1985) et ceux de la vallée d'Ossau dans les Pyrénées atlantiques ou occidentales (Laville \& Vinçon 1986).

Avec 131 espèces récoltées dans la vallée d'Ossau et 167 dans la vallée d'Aure, les Chironomidés sont le groupe d'invertébrés benthiques le plus diversifié des eaux courantes de ces deux vallées; ils représentent $26 \%$ de la biodiversité de la faune macrobenthique. 
Une analyse comparative des communautés chironomidiennes de ces deux systèmes avait débouché sur une zonation altitudinale des 119 espèces les plus fréquentes ou les plus typiques et sur une caractérisation biotypologique commune à ces deux écosystèmes de montagne depuis la zone alpine $(2400 \mathrm{~m})$ jusqu'à la zone piémont (400 m) (Laville \& Vinçon 1991).

La rivière Aude, depuis sa source jusqu'à la ville de Carcassonne ( $\mathrm{km} \mathrm{130)}$, coule du sud au nord dans la partie orientale de la chaine pyrénéenne (Fig.1); elle appartient au réseau méditerranéen français caractérisé par des conditions climatiques et thermiques différentes, avec des températures estivales élevées, des débits irréguliers et des crues violentes en automne.

La démarche suivie sur les $140 \mathrm{~km}$ de son cours supérieur et moyen s'organise autour de deux objectifs :

1) établir un inventaire faunistique des Chironomidés du cours supérieur et moyen d'une rivière des Pyrénées orientales;

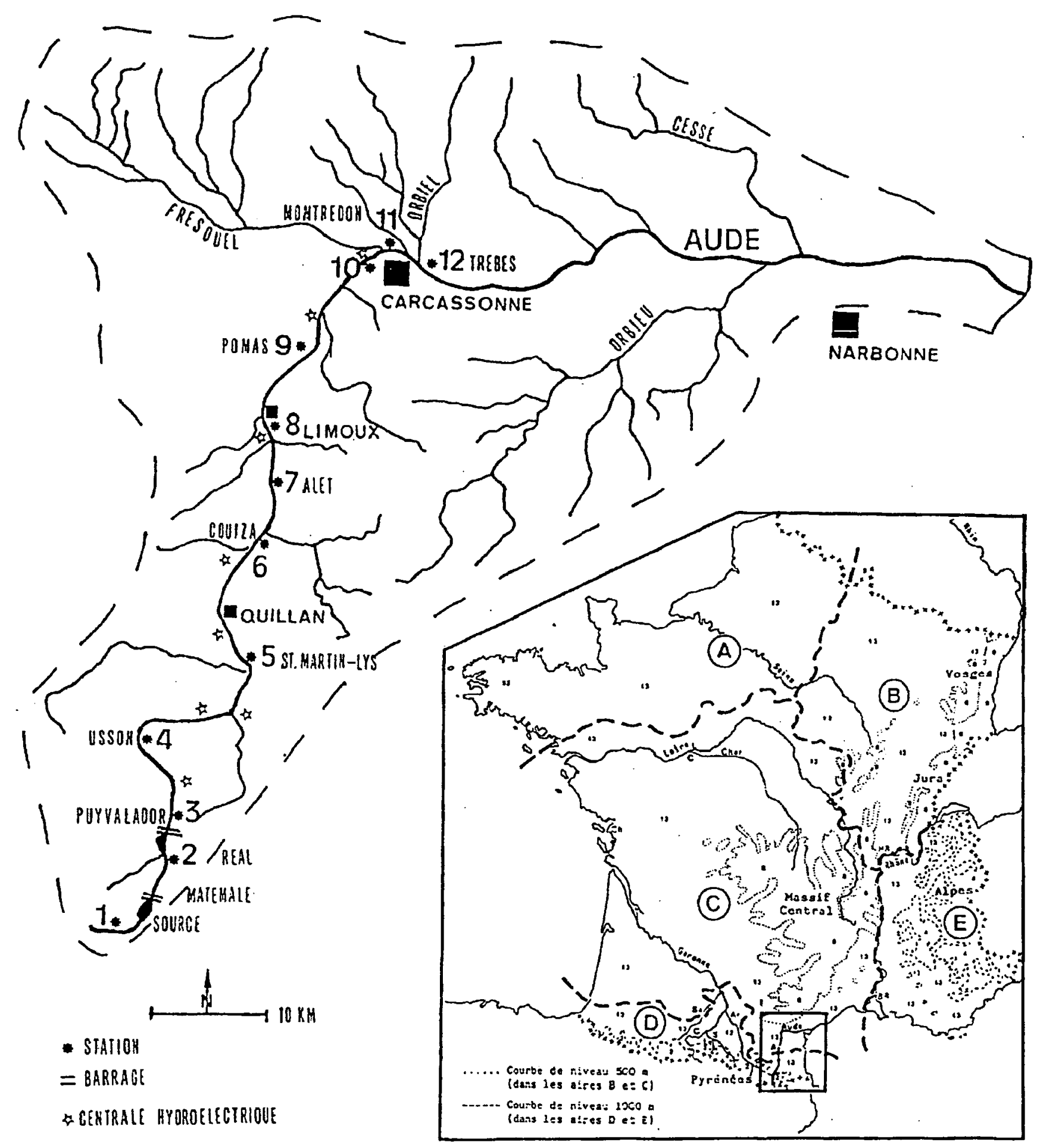

Fig. 1. Réseau et bassin hydrographiques de la rivière Aude. Localisation des 12 stations prospectées. Carte de France d'après Serra-Tosio \& Laville (1991).

Fig. 1. River system and drainage basin of the River Aude. Location of the 12 sites studied. Map of France from Serra-Tosio \& Laville (1991). 
2) analyser la distribution des espèces en relation avec l'altitude et l'habitat qui débouche sur une classification biotypologique basée sur les communautés de Chironomidés.

\section{Présentation de la vallée de l'Aude : choix des stations}

La rivière Aude a été étudiée dans 12 stations échelonnées entre le $\mathrm{km} 5$ et le km 140 (Fig. 1). Les principales caractéristiques morphométriques des 12 stations sont récapitulées sur le tableau 1.

L'Aude, depuis sa source $(2200 \mathrm{~m})$ au pied du mont Carlit (2921 m) traverse successivement la haute chaine granitique et schisteuse, l'avant pays calcaire de Gesse $(\mathrm{km} \mathrm{43)}$ à Quillan $(\mathrm{km} 68)$ dans des gorges profondes du défilé de Pierre Lys et enfin le piémont marneux et mollassique jusqu'à son embouchure au Grau de Vendre, $20 \mathrm{~km}$ à l'est de Narbonne. A Montredon (station 11), $5 \mathrm{~km}$ à l'aval de Carcassonne, elle prend une direction ouest-est. Dans son cours supérieur, la rivière a subi des modifications de ses conditions physiques, notamment un contrôle du débit par les deux barrages de Matemale (1440 m, amont St. 2) et de Puy- valador (1400 m, amont St. 3), ce qui amortit les crues printanières et limite l'étiage estival. Entre Usson $(770 \mathrm{~m}$, St. 4) et Axat (400 m), des sources karstiques assurent la moitié du débit de la rivière et limitent également la sévérité des étiages.

Dans la haute vallée jusqu'à Limoux (St. 8) une pente élevée (moy. $1,4 \%$ ) et un débit suffisant ont été favorables à l'installation de six microcentrales électriques, notamment à Alet et Limoux, qui provoquent une instabilité des débits.

La partie moyenne et inférieure de l'Aude, à l'aval du défilé de Pierre-Lys est soumise à différentes perturbations d'origine industrielle de Quillan à Couiza (St. 6), agricole (vignoble de Limoux, St. 8) et urbaine (Carcassonne : 44000 hab.).

Entre les stations 11 et 12, l'Aude reçoit les eaux fortement enrichies par l'agriculture et les rejets industriels du Fresquel et les eaux de l'Orbiel également enrichies par les industries agroalimentaires de la région du Lauragais. L'amplitude thermique annuelle varie entre $10^{\circ} \mathrm{C}$ à Puyvalador $(1400 \mathrm{~m}, \mathrm{St} .3)$ et $16,5^{\circ} \mathrm{C}$ à Montredon (90 m, St. 11) (Tableau 1).

Tableau 1. Principales caractéristiques morphométriques, géologie, végétation, ordre et amplitude thermique des 12 stations de l'Aude supérieure et moyenne.

Table 1. Main morphometrical characteristics, geology, vegetation, river order and thermal variation in the 12 sites of the River Aude.

\begin{tabular}{|c|c|c|c|c|c|c|c|c|c|c|}
\hline Stations & Ordre & $\begin{array}{c}\text { Altitude } \\
\text { m }\end{array}$ & $\begin{array}{c}\text { Pente } \\
\%\end{array}$ & $\begin{array}{c}\text { Distance ì la } \\
\text { source } \mathbf{k m}\end{array}$ & $\begin{array}{c}\text { Largeur } \\
\text { m }\end{array}$ & $\begin{array}{c}\text { Profondeur } \\
\text { cm }\end{array}$ & $\begin{array}{c}\text { Température } \\
\text { (min. - } \max .) \\
{ }^{\circ} \mathrm{C}\end{array}$ & Géologie & Substrat & $\begin{array}{c}\text { Végétation } \\
\text { Bassin Versant }\end{array}$ \\
\hline 1. Source & 1 & 1673 & 2,5 & 5 & $0,5-1,5$ & $20-30$ & $0-12$ & Granite & Blocs,gravier, sable & $\begin{array}{l}\text { Pin à crochet } \\
\text { Pinus uncinata }\end{array}$ \\
\hline 2. Réal & 2 & 1440 & 1,3 & 19 & 34 & $20-50$ & $2-17$ & Moraine & $\begin{array}{l}\text { Blocs, galets, } \\
\text { macrophytes }\end{array}$ & Prairie \\
\hline 3. Puyvalador & 3 & 1400 & 2,4 & 21 & $1,5-2$ & $50-100$ & 3-13 & Granite & $\begin{array}{l}\text { Blocs, galets, sable, } \\
\text { macrophytes }\end{array}$ & $\begin{array}{l}\text { Bouleau } \\
\text { Betula pendula }\end{array}$ \\
\hline 4. Usson & 3 & 770 & 4,8 & 38 & $5-7$ & $30-50$ & $4,5-17$ & Granite & Blocs, galets, sable & $\begin{array}{l}\text { Hêtre } \\
\text { Fagus svlvatica }\end{array}$ \\
\hline 5. St. Martin-Lys & 4 & 350 & 2,7 & 60 & $5-10$ & $30-100$ & $6-17$ & Calcaire & $\begin{array}{l}\text { Blocs, galets } \\
\text { gravier, sable }\end{array}$ & $\begin{array}{l}\text { Chène pubescent } \\
\text { Quercus pubescens } \\
\text { Hêtre }\end{array}$ \\
\hline 6. Couiza & 4 & 220 & 1,3 & 80 & $25-30$ & $30-100$ & $8-17$ & $\begin{array}{l}\text { Calcaire } \\
\text { Mame }\end{array}$ & Galets, sable, vase & $\begin{array}{l}\text { Vigne } \\
\text { Vitis vinifera }\end{array}$ \\
\hline 7. Alet & 4 & 200 & 0,7 & 95 & $25-30$ & $30-100$ & $8-21$ & $\begin{array}{l}\text { Calcaire } \\
\text { Schiste }\end{array}$ & Galets, sable, vase & Vigne \\
\hline 8. Limoux & 4 & 161 & 0,6 & 106 & $30-40$ & $30-100$ & $7,5-22$ & Molasse & Galets, gravier, sable & Vigne \\
\hline 9. Pomas & 4 & 140 & 0,3 & 119 & $20-30$ & $30-100$ & $7,5-24$ & Molasse & Galets, sable, vase & Vigne \\
\hline 10. Carcassonne & 4 & 97 & 0,1 & 130 & $20-30$ & $30-100$ & $8-23$ & Molasse & Galets, sable, vase & Vigne \\
\hline 11. Montredon & 4 & 90 & 0,1 & 135 & $20-30$ & $50-100$ & $8-24,5$ & Molasse & Galets, sable, vase & $\begin{array}{l}\text { Vigne } \\
\text { Jardins }\end{array}$ \\
\hline 12. Trèbes & 5 & 64 & 0,1 & 140 & $30-40$ & $50-100$ & $9-25$ & Molasse & $\begin{array}{l}\text { Galets, sable, vase, } \\
\text { macrophytes }\end{array}$ & $\begin{array}{l}\text { Vigne } \\
\text { Jardins }\end{array}$ \\
\hline
\end{tabular}




\section{Matériel et méthodes}

Des collections d'exuvies nymphales de Chironomidés ont été effectuées à l'aide de 2 filets à dérive coniques de type Brundin $\left(850 \mathrm{~cm}^{2}\right.$ d'ouverture, $60 \mathrm{~cm}$ de long et $250 \mu \mathrm{m}$ de vide de maille) exposés pendant 30 à 45 minutes depuis les ponts dans les stations basses ou directement sur le substrat dans les stations hautes, peu larges (2-10 m) et plus faciles d'accès.

Les stations 2 à 10 ont été échantillonnées de février à décembre 1989 à 7 dates espacées d'environ 6 semaines (Tableau 2).

La station 1 n'a pu être échantillonnée en hiver, à cause du gel. La station 11 a été prospectée de mars à décembre. La station 12 (Trèbes) n'a pas été échantillonnée en octobre et décembre, l'étiage rendant impossible le maintien des filets en surface et la prospection depuis les berges, largement colonisées par des macrophytes (Phragmites, Typha, Ceratophyllum) s'étant avérée improductive.

Selon les stations et la date de récolte, 45 à 660 exuvies par échantillon ont été triées et identifiées, soit un total de 21189 exuvies pour les 80 collections effectuées dans l'année.

\section{Résultats}

\section{1. Structure des peuplements de Chironomidés : composition spécifique et abondance relative}

125 espèces (ou taxa) ont été recensés dans les douze stations de la rivière Aude prospectées en 1989. Plus de la moitié (64) concerne les Orthocladiinae qui dominent largement le peuplement avec 82 à $98 \%$ des effectifs récoltés dans les stations 1 à 11 (Tableau 3). A Trèbes (St. 12), ils représentent encore $73 \%$ des effectifs suivis par les Tanytarsini (24\%). Les Chironominae sont les plus diversifiées dans les stations inférieures (8-12) avec 13-14 espèces pour la tribu des Chironomini et 7-9 espèces pour celle des Tanytarsini dont la fréquence des effectifs représente entre 9 et $11 \%$ du peuplement.

Le tableau 4 récapitule la liste des 52 espèces les plus fréquentes du peuplement c'est-à-dire celles avec une fréquence $\geq 1 \%$ au moins dans une des douze stations. La majorité des espèces présente une large répartition altitudinale. La fréquence moyenne (M) des espèces dans les 5 stations hautes $(1673-350 \mathrm{~m})$ et dans les 7 stations basses $(220-84 \mathrm{~m})$ donne une première image de leur distribution altitudinale préférentielle dans ce système lotique de montagne (Tableau 4). Parmi les 32 espèces avec une fréquence moyenne totale $\geq 1 \%$ dans l'une ou l'autre zone, $13\left({ }^{*}\right)$ sont davantage localisées dans la partie haute, $9(* *)$ dans la partie basse. Cette analyse globale permet de différencier un peuplement des stations supérieures (1-5) qui correspond à un Ėpi-Métarhithron d'un peuplement des stations inférieures (6-12) qui représente un Hyporhithron-Epipotamon. Les 10 espèces sans préférendum net pour l'une ou l'autre zone sont considérées comme euryzonales et ubiquistes.

Les peuplements mis en évidence par cette analyse globale des données requièrent une analyse spécifique et stationnelle.

La répartition de certaines espèces, notamment leur remontée altitudinale, peut surprendre si on se réfère à

Tableau 2. Dates des récoltes d'exuvies nymphales de Chironomidés effectuées en 1989 dans les 12 stations de l'Aude; nombre moyen $(\overline{\mathrm{N}})$ d'exuvies triées par récolte et nombre total (T) d'exuvies triées par station.

Table 2. Dates of collections of pupal exuviae sampled in the 12 sites of the River Aude. Mean number $(\overline{\mathrm{N}})$ of exuviae sorted in one collection and total number $(\mathrm{T})$ of exuviae sorted in each site.

\begin{tabular}{|c|c|c|c|c|c|c|c|c|c|c|c|c|}
\hline $\begin{array}{r}\text { Statjons } \\
1989\end{array}$ & $\begin{array}{c}\text { Source } \\
1\end{array}$ & $\begin{array}{c}\text { Réal } \\
2\end{array}$ & $\begin{array}{c}\text { Payvalador } \\
3\end{array}$ & $\begin{array}{c}\text { Usson } \\
4\end{array}$ & $\begin{array}{c}\text { St Martin } \\
5\end{array}$ & $\begin{array}{c}\text { Coniza } \\
6\end{array}$ & $\begin{array}{c}\text { Alet } \\
7\end{array}$ & $\begin{array}{c}\text { Limoux } \\
8\end{array}$ & $\begin{array}{c}\text { Pomas } \\
9\end{array}$ & $\begin{array}{c}\text { Caycassonne } \\
10\end{array}$ & $\begin{array}{c}\text { Montredon } \\
11\end{array}$ & $\begin{array}{c}\text { Trabes } \\
12\end{array}$ \\
\hline $22 / 01$ & & & & & & & & & & & & + \\
\hline 1415,02 & & + & + & + & + & + & + & + & + & + & & + \\
\hline $20 / 03$ & & & & & & & & & & & & + \\
\hline $27-28 / 03$ & & + & + & + & + & + & + & + & + & + & + & \\
\hline $17-18 / 05$ & + & + & + & + & + & + & + & + & + & + & + & + \\
\hline $25-26 / 06$ & + & + & + & + & + & + & + & + & + & + & + & + \\
\hline $26-27 / 08$ & + & + & + & + & + & + & + & + & + & + & + & + \\
\hline $14-15 / 10$ & + & + & + & + & + & + & + & + & + & + & + & \\
\hline $16-17 / 12$ & + & + & + & + & + & + & + & + & + & + & + & \\
\hline \multicolumn{13}{|c|}{ Exuvies triees } \\
\hline Moy. $(\bar{N})$ & 45 & 281 & 104 & 117 & 114 & 241 & 232 & 629 & 660 & 370 & 98 & 190 \\
\hline Total (T) & 225 & 1957 & 728 & 819 & 798 & 1687 & 1624 & 4403 & 4620 & 2590 & 588 & 1140 \\
\hline
\end{tabular}


Tableau 3. Richesse spécifique et abondance relative (\%) des effectifs des principales sous-familles ou tribus dans les 12 stations de l'Aude supérieure et moyenne (récoltes 1989).

Table 3. Specific richness, frequency (\%) of the subfamilies and tribes in the 12 sites of the River Aude (1989 collections).

\begin{tabular}{|c|c|c|c|c|c|c|c|c|c|c|c|c|c|c|}
\hline \multirow[t]{2}{*}{ Statlons } & \multirow{2}{*}{$\begin{array}{c}\text { Source } \\
1\end{array}$} & \multirow{2}{*}{$\begin{array}{c}\text { REal } \\
2\end{array}$} & \multirow{2}{*}{$\begin{array}{c}\text { Puyvalador } \\
3\end{array}$} & \multirow{2}{*}{$\begin{array}{c}\text { Usson } \\
4\end{array}$} & \multirow{2}{*}{$\begin{array}{c}\text { St.Martin } \\
5\end{array}$} & \multirow{2}{*}{$\begin{array}{c}\text { Coulza } \\
6\end{array}$} & \multirow[t]{2}{*}{ Alet } & \multirow{2}{*}{$\begin{array}{c}\text { LImoux } \\
8\end{array}$} & \multirow{2}{*}{$\begin{array}{c}\text { Pomas } \\
9\end{array}$} & \multirow{2}{*}{$\begin{array}{c}\text { Carcassonne } \\
10\end{array}$} & \multirow{2}{*}{$\begin{array}{c}\text { Montredon } \\
11\end{array}$} & \multirow{2}{*}{$\begin{array}{c}\text { Trèbes } \\
12\end{array}$} & \multicolumn{2}{|c|}{ Total } \\
\hline & & & & & & & & & & & & & $\mathbf{N}$ & $\$$ \\
\hline \multicolumn{15}{|c|}{ Bachonomyilnae } \\
\hline Espèces & & & & & & & & 1 & 1 & & & & 1 & 0,8 \\
\hline Effectifs $\%$ & & & & & & & & 0,1 & 0,1 & & & & & \\
\hline \multicolumn{15}{|l|}{ Tanypodinae } \\
\hline Especes & 4 & 2 & 2 & 2 & 1 & 1 & 0 & 3 & 2 & 4 & 1 & 2 & 10 & 8,1 \\
\hline Effectifs \% & 6,1 & 0.2 & 0,4 & 0,9 & 0,1 & 0,02 & 0 & 0.7 & 0,1 & 0,4 & 0.3 & 0,5 & & \\
\hline \multicolumn{15}{|l|}{ Dlamesinae } \\
\hline Especes & 0 & 2 & 1 & 3 & 1 & 0 & 1 & 2 & 2 & 0 & 0 & 0 & 6 & 4,8 \\
\hline Efrectifs \% & 0 & 0,1 & 0,6 & 1,6 & 0,1 & 0 & 0,05 & 0,03 & 0,03 & 0 & 0 & 0 & & \\
\hline \multicolumn{15}{|l|}{ Prodiamesinae } \\
\hline Especos & 0 & 2 & 1 & 0 & 1 & 1 . & 0 & 1 & 0 & 0 & 0 & 0 & 2 & 1,6 \\
\hline Effectifs \% & 0 & 0.2 & 0,4 & 0 & 0.1 & 0,1 & 0 & 0,02 & 0 & 0 & 0 & & & \\
\hline & & & & & & & & & & & & 0 & & \\
\hline \multicolumn{15}{|l|}{ Orthocladinae } \\
\hline Especes & 24 & 34 & 38 & 40 & 28 & 35 & 31 & 36 & 34 & 33 & 24 & 28 & 65. & 52,4 \\
\hline Effectifs \% & 82,1 & 95,2 & 93,7 & 96.4 & 96.8 & 97,7 & 95.3 & 88.1 & 89,2 & 89,3 & 84,3 & 73,1 & & \\
\hline \multicolumn{15}{|l|}{ Chironomini } \\
\hline Especes & 2 & 2 & 0 & 1 & 2 & 2 & 3 & 13 & 13 & 14 & 14 & 13 & 26 & 21,0 \\
\hline Efrectifs \% & 1,3 & 0.9 & 0 & 0,3 & 0.8 & 0.1 & 0.2 & 2 & 0.8 & 1,6 & 4.5 & 2,7 & & \\
\hline \multicolumn{15}{|l|}{ Tanytarsini } \\
\hline Especes & 5 & 3 & 5 & 3 & 2 & 6 & 4 & 9 & 9 & 9 & 7 & 7 & 14 & 11,3 \\
\hline Effectifs \% & 10.5 & 3,5 & 5 & 1.2 & 2.1 & 2,1 & 4,9 & 9.2 & 9.9 & 8.6 & 10,9 & 23,9 & & \\
\hline Total espèces & 35 & 45 & 47 & 49 & 35 & 45 & 38 & 64 & 61 & 63 & 46 & 51 & 124 & 100 \\
\hline
\end{tabular}

leur écologie habituelle. C'est notamment le cas de $E$. gracei, espèce plutôt eurytherme de plaine, dont la plus forte abondance se situe dans la station de Réal (St. $2: 1440 \mathrm{~m}$ ). Les caractéristiques mésologiques de cette station située dans une zone de méandres, à faible pente, sur un haut plateau sans végétation riveraine, au substrat riche en macrophytes (Ranunculus aquatilis L.) et Bryophytes, avec des températures estivales de $17^{\circ} \mathrm{C}$ sont une explication à l'ascencion de cette espèce. La remontée de $C$. bicinctus et $C$. tremulus, abondantes dans cette station 2, peut s'expliquer de la même façon ainsi que celle d'espèces moins fréquentes qui colonisent normalement des rivières de plaine : $C$. fuscus, $C$. sylvestris, $N$. rectinervis, $R h$. chalybeatus, $T$. ejuncidus.

L'analyse de la composition spécifique doit être complétée par la liste des espèces considérées «rares» relativement à leur fréquence $<1 \%$ mais qui sont souvent présentes dans plusieurs stations. Cette liste de 73 espèces récoltées en 1989 est récapitulée sur le tableau 5.

24 espèces ont été recensées dans 4 stations ou plus, 49 dans 1 à 3 stations seulement.
Leur rareté apparente ne les exclue pas en tant qu'espèces représentatives d'une zone altitudinale particulière de la rivière. Leur caractère sténoèce permet de les considérer comme espèces indicatrices potentielles d'une zone typologique particulière.

Cette liste des espèces recensées en 1989 a été enrichie par 13 espèces récoltées ultérieurement et que l'on peut classer «rares»: 10 en 1990 à la station 9 (Pomas) lors d'une étude de 2 cycles journaliers d'émergences (Gendron \& Laville 1993) et 3 en 1993 lors d'un suivi annuel de l'impact d'une crue catastrophique dans les stations 6 (Couiza) à 9 (Pomas) (Gendron 1996). Ce complément porte à 138 le total des espèces de Chironomidés recensées à ce jour dans l'Aude supérieure et moyenne.

D'un point de vue faunistique, 14 espèces sont des citations nouvelles pour la Faune de France, et ont été récemment inventoriées (Laville \& Serra-Tosio 1995). 6 d'entre elles $(*)$ sont également des premières citations pour la zone $2(\geq 1000 \mathrm{~m})$ des Pyrénées de la Limnofauna Europaea (Fittkau \& Reiss 1978). 
Tableau 4. Abondance relative (\%) des 52 espèces de Chironomidés les plus fréquentes ( $\geq 1 \%$ dans au moins une station) dans les 12 stations de l'Aude supérieure et moyenne (récoltes 1989).

Table 4. Frequency of the 52 most frequent chironomid species ( $\geq 1 \%$ at least in one site) in the 12 sites of the River Aude (1989 collections).

\begin{tabular}{|c|c|c|c|c|c|c|c|c|c|c|c|c|c|c|}
\hline & \multicolumn{12}{|c|}{ Abondance relattve $g$} & \multicolumn{2}{|c|}{ Prequence moyentre \% } \\
\hline Statlons & 1 & 2 & 3 & 4 & 5 & 6 & 7 & 8 & 9 & 10 & 11 & 12 & St 1.5 & $\mathrm{SL} \quad 6 \cdot 12$ \\
\hline \multicolumn{15}{|l|}{ Tanypodinae (2) } \\
\hline Nilotanypus dubius (Mg.) & 3,5 & & & & & & & & & & & & 0,5 & \\
\hline Zarrelimyia melanura (Mg) & 1,8 & & & & & & & & & & & & 0,4 & \\
\hline \multicolumn{15}{|l|}{ Orthocladilnae (41) } \\
\hline Candiocladius capucinus Zeth. & & + & + & + & 2,1 & + & & & & & & & 0.4 & \\
\hline * Cardiocladius fuseus K & & + & & + & & + & + & 2 & 1,5 & 2,8 & 3,1 & 1.1 & + & 1,5 \\
\hline Cricotopus annulator $\mathrm{G}$. & 1,3 & + & 2.9 & 1.6 & & 4 & 2,7 & 10,9 & 12,4 & 7 & 4,6 & 7,5 & 1,2 & 7 \\
\hline Cricotopus bicinctus (Mg.) & 1,8 & 5.4 & & & & + & + & + & + & 4,8 & 3,1 & 17.9 & 1,4 & 3,7 \\
\hline Cricotopus curtus Hiv. & 2,7 & + & & & & & & & & & & & 0,5 & \\
\hline Cricotopus similis $\mathbf{G}$. & & & 1 & 2 & + & 4,2 & + & & + & + & + & + & 0,6 & 0.6 \\
\hline Cricotopus stlvestris (Fabr.) & & + & & & & + & 2.6 & + & + & + & + & + & + & 0.4 \\
\hline - Cricotopus vemulus (L) & & 5.9 & + & 1,3 & + & 1 & + & + & + & + & + & + & 1,4 & + \\
\hline Cricotopus crifascia Edw. & & & & + & + & + & + & + & + & 1,1 & + & + & + & 0.2 \\
\hline ** CHcosopus vierriensis G. & & & & & & + & 2.2 & 4,1 & 13.2 & 10,8 & 14,1 & 2,3 & & 6.7 \\
\hline Eutiefferiella chypeata (K) & + & 9.6 & 9,7 & 10 & + & + & + & 1,5 & + & & & & 5,9 & 0,3 \\
\hline - Eutiefferiella coerulescens (K.) & 4,9 & + & 1,9 & 1,1 & + & & & & & & & & 1.6 & \\
\hline Eukiefferiella fithaui Lehm. & & & 2,9 & + & 1,1 & & & & & & & & 0,8 & \\
\hline Eukiefferiella fuldensis Lehm. & & & + & 1,6 & & & & & & & & & 0,3 & \\
\hline - Eukiefferiella gracei (Edw.) & & 7,1 & + & & & 1 & + & + & + & 1,6 & + & + & 1.4 & 0.2 \\
\hline Eukiefferiella ilkleyensis (Edw.) & & 8,8 & 6,1 & 1,3 & 19,2 & 19.1 & 17,2 & 8.7 & 2,2 & 14,1 & 6,8 & 4 & 7,1 & 10,3 \\
\hline "Eukiefferiella lobifena G. & 4.4 & 2.3 & 4,4 & 3,9 & 1 & + & + & + & + & + & & & 3,2 & + \\
\hline - Eukiefferlella similis $\mathrm{G}$. & + & + & 1,1 & 5.8 & 14 & + & + & + & & & & & 4,2 & + \\
\hline * Heleniella omaticollis Edw. & 4,9 & & + & + & + & & & & & & & & 1 & \\
\hline Heterotaryecorsus apicalis K. & 1,3 & + & + & & & & & & & & & & 0,3 & \\
\hline Herrobrissocladius mancidus (Walk) & 4,4 & + & + & + & & & & & & & & & 0,9 & \\
\hline Nanocladius bicolor Zett & & & & & & & & 1 & + & 1.7 & 2.6 & 3.5 & & 1,8 \\
\hline Nanocladius rectinervis K & & + & + & & 1.2 & + & & + & 1,3 & 1,1 & 1 & 2.2 & 0.2 & 0,8 \\
\hline - Orhacladius ashei Sop. & & 1.5 & 3,6 & 4,3 & 4,8 & + & + & + & + & + & + & + & 2,8 & + \\
\hline - Orhocladius frigidus Zett. & & 2.4 & 6.8 & 9,8 & 2.4 & + & + & + & & & & & 4,3 & + \\
\hline * Orhockadius oblidens (Walk) & & + & + & + & + & 9,9 & 4,6 & 9,1 & 2,6 & 4,1 & 5,5 & 6,6 & + & 6,1 \\
\hline Onhociadius rivicala $\mathrm{K}$. & & 2,9 & 1,3 & 4,9 & 7,3 & 1.7 & 1 & 1,2 & + & 1,3 & + & 1.1 & 3.3 & 0,9 \\
\hline Orthaciadius rivulorum $\mathrm{K}$. & & + & 1,7 & + & + & + & & + & + & + & & & 0,3 & + \\
\hline Orthocladius nubicundus (Mg) & & 6,7 & 3,6 & 11,8 & 13,1 & 11 & 9,3 & 7,3 & 1,3 & 2,5 & 2,7 & + & 7 & 4,9 \\
\hline * Orthocladius saxosus (Tok) & & + & 1,7 & 4.7 & & & & & & & & & 1,3 & \\
\hline Orthocladius vaillansi Lang. \& Crans. & & & & + & 4,1 & + & + & & & & & & 0,8 & + \\
\hline Parnacticotopus niger (K.) & & & + & + & 7,6 & 5 & 3,5 & + & + & + & + & + & 1,5 & 1.2 \\
\hline Paramerriacnemus stylatus (K.) & 1,8 & + & 1,3 & 6,3 & + & + & + & + & + & + & & + & 1,9 & + \\
\hline Paraurichociladius nufiventris (Mg.) & 1,3 & 2,2 & 5,1 & 1.8 & 5,2 & 23 & 30,7 & 7,8 & 5,3 & 15,4 & 11.4 & 9,7 & 3,2 & 14,8 \\
\hline - Paratrichocladius skirwithensis (Edw.) & + & + & 3,2 & 2,4 & & & & & & & & & 1,1 & \\
\hline Paratrissocladius excerptus (Walk) & & & & 1,1 & & & & & & & & & 0,2 & \\
\hline - Parorthocladius nudipennis (K.) & & & 1,9 & 4,1 & & & & & & & & & 1,2 & \\
\hline * Rheocricotopus chalybeatus (Edw.) & & + & & & + & + & & + & 1.2 & 2,8 & 13,5 & + & + & 2,5 \\
\hline * Synorthociadius semlvirens (K.) & + & + & + & 2,8 & & 1.4 & 1,7 & 11.7 & 3,8 & 1.5 & 4,6 & 8 & 0.6 & 4,7 \\
\hline Thienemanniella vinata Edw. & 35 & $\mathbf{3 4 , 3}$ & + & 2 & + & 1,3 & 2,3 & 11,6 & 38,4 & 10,4 & 3,6 & 3.2 & 14,3 & 10.1 \\
\hline Tvelersia calvescens (Edw.) & 10,6 & 2 & 25,5 & 4,6 & 7,9 & 14,4 & 9 & 3.4 & 1,5 & 4 & 4,8 & 2.1 & 10.1 & 5.6 \\
\hline \multicolumn{15}{|l|}{ Tagrtarsidal (9) } \\
\hline Micropsectra atrojasciata (K.) & + & 3,1 & 2,3 & + & + & + & + & + & 1,8 & 1,4 & 2,7 & 13 & 1.1 & 2.7 \\
\hline Micropsectra bidentauc $\mathrm{G}$. & + & & 1 & + & & & & & & & & & 0.2 & \\
\hline ** Neozavrelia fuldensis Fitt. & & & + & + & 1.3 & 1.1 & 3,3 & 3,7 & 1,3 & 2.1 & + & & 0.3 & 1.5 \\
\hline * Paratanytarsus dissimilis Joh. & & & & & & & & + & + & + & 1.9 & 5,3 & & 1 \\
\hline Rheotanyzarsus penzapoda $\mathrm{K}$. & & + & 1,3 & & & & & & & & & & 0,3 & . \\
\hline ** Rheotanytarsus rhenanus Klink. & & & & & & + & 1,3 & + & 5 & 2 & 1,9 & + & & 1.5 \\
\hline - Stempellina brevis (Edw.) & 7.5 & & & & & & & & & & & & 1.5 & \\
\hline Tanytarsus brundini Lina. & & & & & & + & & + & + & + & 1,9 & & & 0,3 \\
\hline ** Tanytarsus ejuncidus (Walk.) & 1,8 & + & & & & + & + & 3,2 & + & 1,7 & 2,9 & 1,9 & 0.4 & 1.4 \\
\hline
\end{tabular}

* Eukiefferiella ancyla Limnophyes edwardsi

* Orthocladius (Euorth.) ashei

* Orthocladius (s. str.) carlatus

* Orthocladius (s. str.) pedestris

* Orthocladius (s. str.) ruffoi Orthocladius (s. str.) vaillanti

* Parachaetocladius abnobeus Parakiefferiella fennica

7 espèces, déjà signalées en France où à des altitudes inférieures à $1000 \mathrm{~m}$ dans les Pyrénées $\left(^{\circ}\right.$ ) (Laville \& Vinçon 1986) sont également nouvelles pour la zone $2 \mathrm{de}$ la Limnofauna.
Chironomus nuditarsis Cryptochironomus obreptans

Robackia demeijerei

Cladotanytarsus pallidus

Stempellina almi 
Tableau 5. Liste des 73 espèces peu fréquentes ou rares et leurs stations de récoltes en 1989. 10 espèces $(*)$ récoltées à Pomas (St. 9) en 1990 et 3 espèces (**) récoltées dans les stations 6 à 9 en 1993 complètent l'inventaire faunistique des Chironomidés de la rivière Aude.

Table 5. List of the 73 infrequent or rare species with their sampling sites in 1989.10 species $\left(^{*}\right)$ collected at the site 9 (Pomas) in 1990 and 3 species $(* *)$ collected at the site 6 to 9 in 1993 enlarge to 138 the chironomid inventory of the River Aude.

\section{Espèces}

\section{Stations}

\begin{tabular}{|c|c|c|c|c|c|c|c|c|c|c|c|c|}
\hline & 1 & 2 & 3 & 4 & 5 & 6 & 7 & 8 & 9 & 10 & 11 & 12 \\
\hline \multicolumn{13}{|l|}{ Tanypodinae (11) } \\
\hline ** Ablasbesmyia longistyla Fitt. & & & & & . & & $\mathrm{x}$ & & & & & \\
\hline * Ablasbesmyia monilis (L.) & & & & & & & & & $\mathrm{x}$ & & & \\
\hline Conchapelopia melanops (Mg.) & & $x$ & $\mathbf{x}$ & & $\mathrm{x}$ & & & & & & & \\
\hline Macropelopia nebulosa (Mg.) & & & $\bar{x}$ & $x$ & & & & & & & & \\
\hline Procladius choreus $(\mathrm{Mg})$. & & & & & & & & & $\mathrm{x}$ & $\mathrm{x}$ & & \\
\hline Procladius sagittalis (K.) & $x$ & & & & & $\mathbf{x}$ & & $\mathbf{x}$ & $\bar{x}$ & $\bar{x}$ & $\mathbf{x}$ & $x$ \\
\hline Rheopelopia ornata (Mg.) & & & & & & & & & & $\mathbf{x}$ & & \\
\hline Tanypus punctipennis (Mg.) & & & & & & & & $x$ & & $\mathbf{x}$ & & $\mathbf{x}$ \\
\hline Thienemannimyia geijskesi (G.) & & $\mathrm{x}$ & & & & & & & & & & \\
\hline Thienemannimyia lentiginosa (Fries) & $\mathbf{x}$ & & & & & & & $\mathbf{x}$ & & & & \\
\hline Thienemannimyia northumbrica (Edw.) & & & & & & & & & $\mathrm{x}$ & & & \\
\hline \multicolumn{13}{|l|}{ Buchonomyiinae (1) } \\
\hline Buchonomyia thienemanni Fitt. & & & & & & & & $\mathbf{x}$ & $\underline{x}$ & & & \\
\hline \multicolumn{13}{|l|}{ Diamesinae $(6)$} \\
\hline Boreoheptagyia legeri $(\mathrm{G})$. & & & & $\mathbf{x}$ & & & & & & & & \\
\hline Diamesa insignipes $\mathrm{K}$ & & $\mathbf{x}$ & & & & & & & & & & \\
\hline Diamesa tonsa (Hal, sensu $\mathrm{Pag}$ ) & & & $\mathbf{x}$ & $\mathbf{x}$ & $\mathbf{x}$ & & & & & & & \\
\hline Potthastia gaedii (Mg.) & & & & & & & & & $\mathrm{x}$ & $\mathbf{x}$ & & \\
\hline Potthostia longimanus $\mathrm{K}$. & & & & & & & & $\mathbf{x}$ & $\mathrm{x}$ & $\mathbf{x}$ & $\mathbf{x}$ & \\
\hline Pseudodiamesu branickii (Now.) & & $\mathrm{x}$ & & $\mathbf{x}$ & & & & & & & & \\
\hline \multicolumn{13}{|l|}{ Prodiamesinae (2) } \\
\hline Odontomesa fulva $(\mathrm{K})$ & & $x$ & & & $\mathbf{x}$ & & & & & & & \\
\hline Prodiamesa olivacea (Mg.) & & $\mathrm{x}$ & $\mathbf{x}$ & & & $\mathbf{x}$ & & $\mathbf{x}$ & & & & \\
\hline \multicolumn{13}{|l|}{ Orthocladiinae $(30)$} \\
\hline Brillia modesta (Mg.) & $\mathbf{x}$ & & $\mathrm{x}$ & & & & & & & & & \\
\hline Brillia flavifrons Johannsen & $\mathbf{x}$ & & & $\mathbf{x}$ & & & & $\mathbf{x}$ & $\mathbf{x}$ & $\mathbf{x}$ & & $\mathbf{x}$ \\
\hline Corynoneura scutellata Winn. & $x$ & & & & & & & & $\mathbf{x}$ & & & \\
\hline Cricotopus triannulatus (Macg.) & & . & & & & & & & $\mathbf{x}$ & & & \\
\hline Eukiefferiella ancyla Svensson & & & $\mathbf{x}$ & $\mathbf{x}$ & $x$ & $\mathbf{x}$ & & & & & & \\
\hline ** Eukiefferiella claripennis (Lundb.) & & & & & & $\mathbf{x}$ & & $\mathbf{x}$ & $\mathrm{x}$ & & & \\
\hline * Limnophyes edwardsi Saether & & & & & & & & & $x$ & & & \\
\hline Limnophyes natalensis $(\mathrm{K})$. & $\mathbf{x}$ & & & & & & $x$ & $\mathbf{x}$ & $\mathbf{x}$ & & & $\mathbf{x}$ \\
\hline Metriocnemus eurynotus (Holmg.) & & & & & & & & & & $\mathrm{x}$ & & \\
\hline * Nanocladius balticus Pal. & & & & & & & & $\mathbf{x}$ & & & & \\
\hline Nanocladius parvulus (K.) & & & & $x$ & & & & & & & & \\
\hline Orthocladius obumtratus Johannsen & & & & $\mathrm{x}$ & & $\mathrm{x}$ & & & & & & \\
\hline Orthocladius fuscimanus $(\mathbf{K})$ & & & & $x$ & $\mathbf{x}$ & $\mathbf{x}$ & $\mathbf{x}$ & $\mathbf{x}$ & $\underline{x}$ & $\mathrm{x}$ & $\mathbf{x}$ & $\mathbf{x}$ \\
\hline Orthocladius of carlatus Roback & $\mathrm{x}$ & $\mathrm{x}$ & & & & $\mathrm{x}$ & $x$ & $\mathbf{x}$ & $x$ & $x$ & & \\
\hline Orthocladius wetterensis $\mathrm{Br}$. & & & $\mathbf{x}$ & & & & & & & & & \\
\hline Orthocladius pedestris $\mathbf{K}$ & & & $\bar{x}$ & & & & & & & & & \\
\hline Orthocladius ruffoi Rossaro & $\mathbf{x}$ & $\mathrm{x}$ & $\mathbf{x}$ & $\mathbf{x}$ & $\mathbf{x}$ & $\mathbf{x}$ & & & & & & \\
\hline Parachaeotocladius abnobaeus (Wullk) & & & $\mathbf{x}$ & & & & & & & & & \\
\hline Paracladius conversus (Walk.) & & $\mathrm{x}$ & & & & $\mathbf{x}$ & $\mathbf{x}$ & $\mathbf{x}$ & $\mathbf{x}$ & $\mathrm{x}$ & $\mathbf{x}$ & $\mathbf{x}$ \\
\hline Parakiefferiella bathophila (K.) & & - & & & & $\mathbf{x}$ & $\mathrm{x}$ & $\mathbf{x}$ & & & & \\
\hline * Parakiefferiella fennica Tuiskunen & & & & & & & & & $\mathbf{x}$ & & & \\
\hline Paraphaenocladius irritus (Walk.) & & & & & & & & & & $\underline{x}$ & & \\
\hline Pseudosmittia oxoniana (Fdw.) & $\mathrm{x}$ & & & & $x$ & & $\mathbf{x}$ & $x$ & & $\mathbf{x}$ & & \\
\hline Rheocricotopus atripes (K.) & & $\mathbf{x}$ & & & & & & & & & & \\
\hline Rheocricotopus fuscipes $(\mathrm{K}$ ) & $\mathbf{x}$ & & $\mathbf{x}$ & & & & & & & & & \\
\hline Symbiocladius rhithrogenae (Zav.) & & & & $\mathbf{x}$ & & & & & & & & \\
\hline Thienemanniella acuticornis $(\mathrm{K}$.) & & & & $\mathbf{x}$ & & & & & & & & \\
\hline * Thienemanniella majuscula (Edw.) & & & & & & & & & $x$ & & & \\
\hline * Thienemanniella Pe 2a (Langton 91) & & & & & & & & & $\mathbf{x}$ & & & \\
\hline Thienemannielka Pe 4 (Langton 91) & & & & & & & & & & $\mathbf{x}$ & & \\
\hline
\end{tabular}


Tableau 5. Suite.

Table 5. Continued.

\begin{tabular}{|c|c|c|c|c|c|c|c|c|c|c|c|c|}
\hline Chironomini (29) & 1 & 2 & 3 & 4 & 5 & 6 & 7 & 8 & 9 & 10 & 11 & 12 \\
\hline Chironomus bernensis Wulk. \& Kldez & & & & & & & & & & & $x$ & \\
\hline Chironomus cingulatus (Mg.) & & & & & & $x$ & & $x$ & $x$ & $x$ & $x$ & $x$ \\
\hline Chironomus luridus Str. & & & & & & $x$ & & & & & & \\
\hline Chironomus nuditarsis Keyl. & & & & & & & & & $\mathbf{x}$ & & & $x$ \\
\hline Chironomus Pel7 (Langton 95) & & & & & & & & & & $\mathbf{x}$ & & \\
\hline * Clapadelma viridula (L.) & & & & & & & & & $\mathbf{x}$ & & & \\
\hline Cryptochironomus obreptans (Walk) & & & & & & & & $\mathrm{x}$ & & & & $x$ \\
\hline * Coyptochironomus rostratus $\mathrm{K}$. & & & & & & & & & $x$ & & & \\
\hline Cryptochironomus Pe1 (Langton 91) & & & & & & & & & & $x$ & & \\
\hline Cryptotendipes pseudotener (G.) & & & & & & & & $\mathbf{x}$ & $x$ & $x$ & $\mathbf{x}$ & $\underline{x}$ \\
\hline Dicrotendipes nervosus (Staeg.) & & & & & & & & $\underline{x}$ & $x$ & $x$ & $x$ & $x$ \\
\hline Dicrotendipes notatus (Mg.) & & & & & & & & & & & & $x$ \\
\hline Glyptotendipes pallens (Mg.) & & & & & & & & & & & $x$ & $x$ \\
\hline Harnischia cf. currilamellatus (Mall.) & $\mathbf{x}$ & & & & & & & $\underline{x}$ & & $x$ & $\underline{x}$ & \\
\hline Microtendipes confinis (Mg.) & & & & & & & $x$ & $x$ & $\bar{x}$ & $\mathrm{x}$ & $\mathrm{x}$ & $x$ \\
\hline Parachironomus arcuatus (G.) & & & & & & & & & & $\mathrm{x}$ & $x$ & $x$ \\
\hline Paracladopelma camptolabis $(\mathrm{K}$ ) & & & & & & & & $x$ & $\bar{x}$ & $x$ & & \\
\hline Paratendipes albimanus (Mg.) & & & & & & & $\mathbf{x}$ & $x$ & $\bar{x}$ & $x$ & 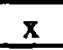 & \\
\hline Phaenopsectra flavipes (Mg.) & & & & & & & & $\mathrm{x}$ & $x$ & & $x$ & $x$ \\
\hline Polypedilum convictum (Walk) & & $\mathrm{x}$ & & $\mathbf{x}$ & $x$ & & & $x$ & & & & \\
\hline Polypedilum cultellatum G. & & & & & $\bar{x}$ & & & $x$ & $\mathbf{x}$ & $\mathbf{x}$ & $x$ & $x$ \\
\hline Polypedilum laetum (Mg.) & & $x$ & & & & & & & & & $x$ & \\
\hline Polypedilum nubeculosum (Mg.) & & & & & & & & & $x$ & & & \\
\hline Polypedilum pedestre $(\mathrm{Mg})$. & $x$ & & & & & & & & $\mathbf{x}$ & & & \\
\hline Polypedilum cf. scalaenum (Schr.) & & & & & & & & $\mathrm{x}$ & $\bar{x}$ & $\bar{x}$ & $\bar{x}$ & $x$ \\
\hline Polypedilum $\mathrm{Pe} 1$ (I angton 91) & & & & & & & & $x$ & & $x$ & & \\
\hline ** Robackia demeijerei (Krus.) & & & & & & $x$ & & & & & & \\
\hline Stenochironomus gibbus (Fabr.) & & & & & & & & & $x$ & & & \\
\hline Stictochironomus histrio (Fabr.) & & & & & & & & & & $x$ & & $\mathbf{x}$ \\
\hline \multicolumn{13}{|l|}{ Tanytarsini (7) } \\
\hline * Cladotanytarsus pallidus $\mathrm{K}$ & & & & & & & & & $\mathbf{x}$ & & & \\
\hline Micropsectra junci (Mg.) & & & $\mathbf{x}$ & & & & & & & & & \\
\hline Paratanytarsus inopertus (Walk) & & & & & & & & $x$ & $\mathrm{x}$ & $\mathrm{x}$ & $\mathbf{x}$ & $x$ \\
\hline * Stempellina almi $\mathrm{Br}$. & & & & & & & & & $x$ & & & \\
\hline Stempellina bausei $(\mathbf{K})$ & $x$ & & & & & & & & & & & \\
\hline Tanytarsus pallidicomis (Walk) & & & & & & $\mathbf{x}$ & & $x$ & $x$ & $\mathrm{x}$ & $x$ & \\
\hline Virgatanytarsus arduennensis (G.) & & & & & & & & $x$ & $x$ & $x$ & $x$ & \\
\hline
\end{tabular}

- l'une pour établir les affinités cénotiques entre les 12 stations;

- l'autre pour rechercher les affinités entre les espèces selon leur répartition altitudinale et leur habitat.

Ces deux analyses ont pour but d'établir une biotypologie des cours supérieur et moyen de la rivière Aude.

\section{2. 1. Essai de zonation}

La première classification qui repose sur les affinités cénotiques entre les 12 stations ne tient compte que des 52 espèces fréquentes $(\geq 1 \%)$ dans au moins une station (Tableau 4). Ces affinités entre les stations sont représentées à l'aide d'un arbre hiérarchique ou dendo- gramme dont l'interprétation mathématique se réfère à Lebart et al. (1982) (Fig. 2).

Pour regrouper les stations nous utilisons comme critère une distance euclidienne de 8 , soit $73 \%$ de la distance maximale entre deux stations (ex. Usson (St. 4) et Montredon (St. 11)).

Cette analyse fait ressortir quatre zones :

- la zone 1 isole la station 1 proche de la source dont la biocénose correspond à un crénon;

- la zone 2 réunit les stations 2-3-4 (Réal, Puyvalador, Usson) avec une biocénose assimilable à un épirhithron; 

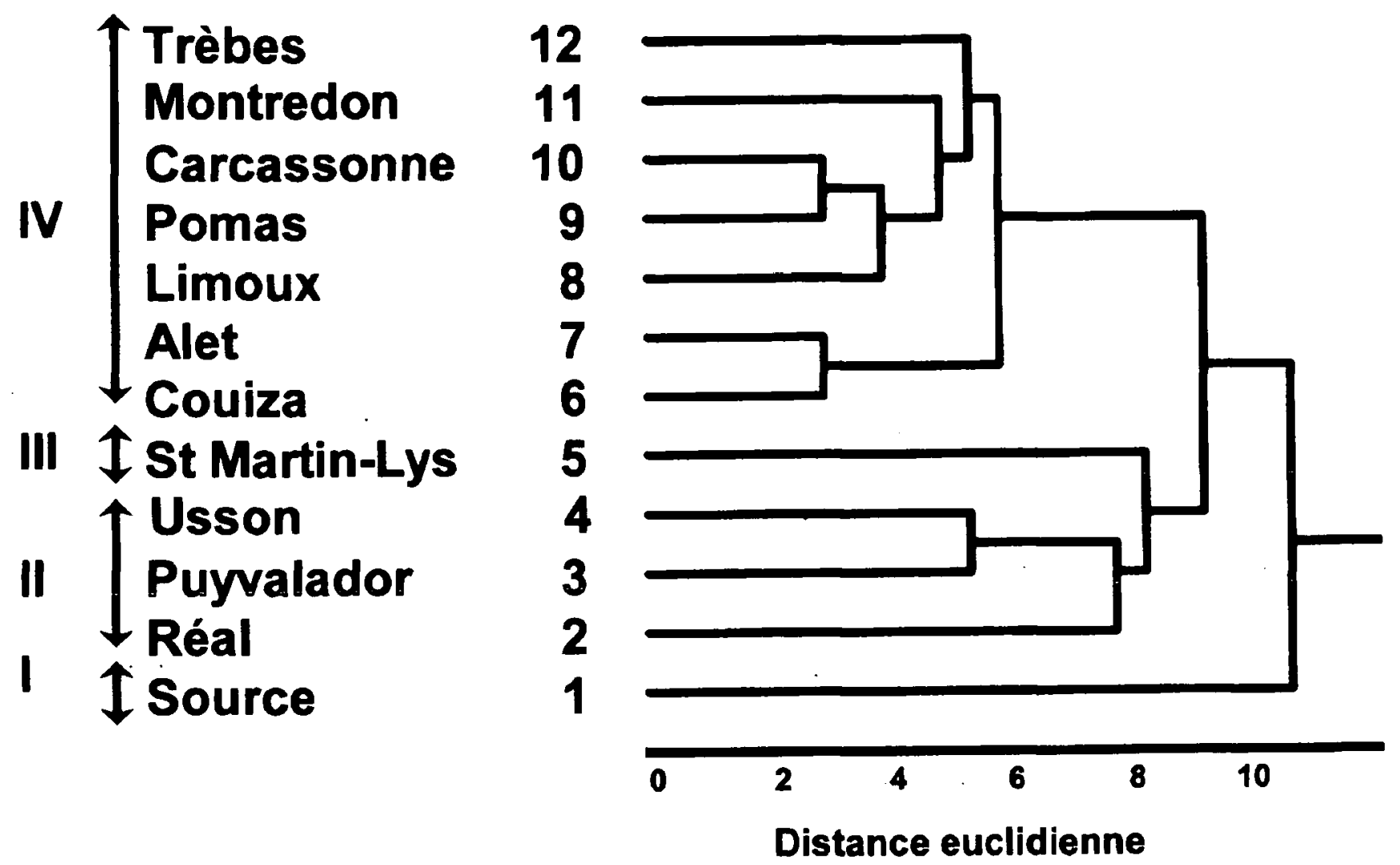

Fig. 2. Dendogramme obtenu à partir de la classification hiérarchique ascendante des 12 stations de l'Aude selon leurs affinités cénotiques : mise en évidence de 4 zones typologiques.

Fig. 2. Dendogram obtained with a cluster analysis of the 12 sites of the River Aude according to their biocenotical affinities displaying four typological zones.

- la zone 3 isole la station 5 (St Martin-Lys) dont la biocénose pourrait correspondre à un métarhithron;

- la zone 4 regroupe les 7 stations de la moyenne vallée, de Couiza $(220 \mathrm{~m})$ à Trèbes $(84 \mathrm{~m})$ dont la biocénose correspond à une association d'espèces de l'hyporhithron et de l'épipotamon.

\section{2. 2. Répartition altitudinale des espèces}

La seconde classification hiérarchique, basée sur la même matrice de données (Tableau 4) regroupe les espèces dont l'affinité traduit une zonation altitudinale semblable (Fig. 3).

5 groupes d'espèces affines se différencient :

- le groupe A : 8 espèces à large répartition altitudinale $(1700-80 \mathrm{~m})$, isolées par le premier noeud sont des espèces euryzonales;

- le groupe $B$ : 13 espèces surtout fréquentes dans les stations basses $(220-80 \mathrm{~m})$, isolées par le deuxième noeud, sont des espèces de plaine;

- le groupe $C: 9$ espèces isolées par le troisième noeud ont une large répartition altitudinale $(1700-400 \mathrm{~m})$ mais plutôt rares en plaine au dessous de $400 \mathrm{~m}$; ce sont des espèces euryzonales de montagne selon Ward (1986);
- le groupe D : 3 espèces de piémont ou de basse montagne $(200-400 \mathrm{~m})$ sont isolées par le quatrième noeud;

- le groupe $\mathrm{E}: 19$ espèces fréquentes dans l'Aude supérieure (700-1700 m) et regroupées par le cinquième noeud peuvent être considérées comme des espèces relativement psychrosténothermes de haute et moyenne montagne.

\section{2. 3. Caractérisation des niveaux typologiques}

Le tableau 6 récapitule l'abondance relative des 5 groupes d'espèces : euryzonales $(A)$, de plaine $(B)$, euryzonales de montagne (C), de piémont (D) de haute et moyenne montagne (E) dans les quatre zones de la rivière Aude définies antérieurement ( $\$ 4$. 2. 1.).

- Les espèces euryzonales (A) dominent dans les 4 zones avec 45 à $64 \%$ du peuplement;

- les espèces de plaine (B) sont surtout fréquentes dans l'hyporhithral (25\%);

- les espèces euryzonales de montagne $(\mathrm{C})$ représentent le tiers des peuplements de l'épirhithral $(35 \%)$ et du métarhithral (33\%);

- les espèces de piémont ou de basse montagne (D) représentent $10 \%$ du peuplement du métarhithral; 


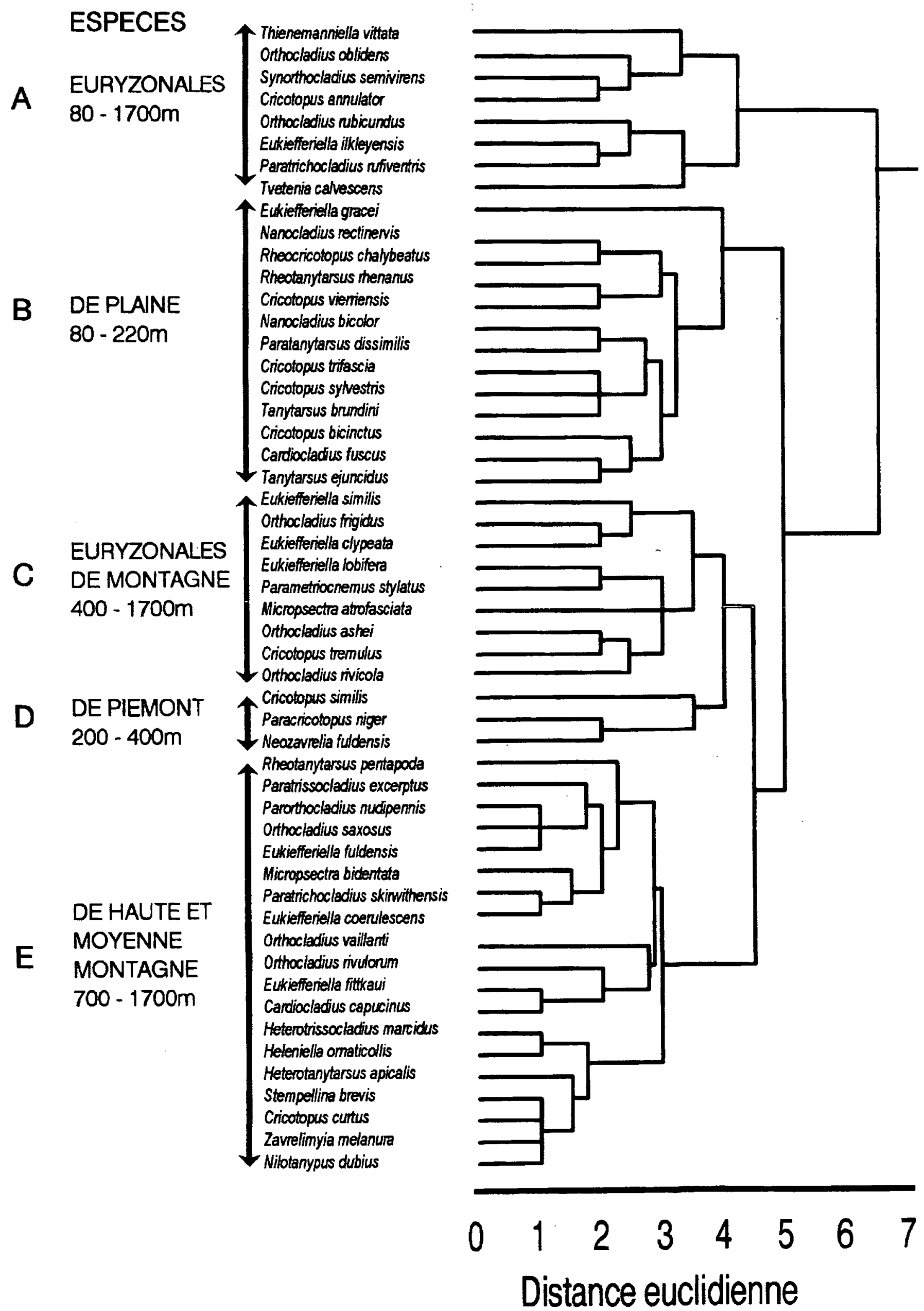

Fig. 3. Dendogramme de la classification hiérarchique des 52 espèces les plus fréquentes $(\geq 1 \%)$ dans les 12 stations de la haute et moyenne rivière Aude mettant en évidence 5 groupements d'espèces en fonction de leur répartition altitudinale.

Fig. 3. Dendogram obtained with a cluster analysis of the 52 most frequent $(\geq 1 \%)$ species of the River Aude displaying 5 species groups according to their altitudinal distribution. 
Tableau 6. Abondance relative (\%) des 5 groupes d'espèces (A, B, C, D, E) de Chironomidés mis en évidence (cf. Fig. 3) dans les 4 zones typologiques (cf. Fig. 2) de la rivière Aude.

Table 6. Frequency of the five species groups (A, B, C, D, E) of Chironomidae displaying (cf. Fig. 3) in four typological zones (cf. Fig. 2) of the River Aude.

\begin{tabular}{|c|c|c|c|c|c|c|c|c|c|}
\hline Zone & Typologie & Ordre & $\mathrm{T}^{\circ} \mathrm{C}$ max. & $\begin{array}{c}\text { A } \\
\text { Euryzonales } \\
80-1700 \mathrm{~m} \\
\end{array}$ & $\begin{aligned} & B \\
& \text { De } \text { plaine } \\
& 80-220 \mathrm{~m} \\
& 80\end{aligned}$ & $\begin{array}{c}\text { C } \\
\text { Euryzonales de } \\
\text { montagne } \\
400-1700 \mathrm{~m} \\
\end{array}$ & $\begin{array}{l}\text { De } \\
200 \\
\end{array}$ & $\begin{array}{l}\mathrm{D} \\
\text { piémont } \\
.400 \mathrm{~m} \\
\end{array}$ & $\begin{array}{c}\mathrm{E} \\
\text { Haute et moyenne } \\
\text { montagne } \\
700-1700 \mathrm{~m} \\
\end{array}$ \\
\hline 1 & Crénal & 1 & 12 & 53 & 4 & 9 & & - & 34 \\
\hline 2 & Epirhithral & $2-3$ & $13-17$ & 45 & 5 & 35 & & 1 & 14 \\
\hline 3 & Métarhithral & 4 & 17 & 47 & 1 & 33 & & 10 & 9 \\
\hline 4 & $\begin{array}{l}\text { Hyporhithral } \\
\text { Epipotamal }\end{array}$ & $4-5$ & $21-25$ & 64 & 25 & 7 & & 4 & - \\
\hline
\end{tabular}

- les espèces de haute et moyenne montagne $(E)$ sont surtout fréquentes près de la source $(34 \%)$ et dans l'épirhithral (14\%).

Ces 4 zones typologiques successives peuvent être définies à la fois par plusieurs espèces soit caractéristiques d'une zone, soit préférentes, c'est-à-dire plus particulièrement abondantes dans une zone qui constitue son préférendum écologique.

* La zone 1, représentée par la seule station 1 $(1673 \mathrm{~m})$, est assimilable à un hypocrénon distant de $5 \mathrm{~km}$ de la source véritable de l'Aude. Elle est située dans l'étage subalpin et les températures maxima ne dépassent pas $12^{\circ} \mathrm{C}$.

Cinq espèces sont caractéristiques ou préférentes :

Zavrelimyia melanura, Heleniella ornaticollis, $\mathrm{He}$ terotanytarsus apicalis, Heterotrissocladius marcidus, Stempellinella brevis.

L'absence de Diamesinae et la présence de Z. melanura et $H$. marcidus, espèces caractéristiques des lacs de la zone alpine (2100-2400 m) des Pyrénées centrales (Laville 1972) soulignent le caractère limnocrène de cette station 1 .

* La zone 2, assimilable à un épirhithron, regroupe les stations 2 et $3(1440-1400 \mathrm{~m})$ d'ordre 2 et la station $4(770 \mathrm{~m})$ d'ordre 3 , situées dans l'étage montagnard. Les températures maxima varient de $13^{\circ}$ à $17^{\circ} \mathrm{C}$ suivant l'altitude. Les 10 espèces caractéristiques ou préférentes de cette zone sont des formes plutôt rhéophiles et oxybiontes :

Eukiefferiella clypeata, E. fittkaui, E. fuldensis, E. gracei, Orthocladius (Euorthocladius) saxosus, Orthocladius (s.str.) frigidus, Paratrichocladius skirwithensis, Parorthocladius nudipennis, Micropsectra bidentata, Rheotanytarsus pentapoda.
Deux espèces, plus rares et psychrosténothermes, peuvent également caractériser cette zone :

\section{Diamesa tonsa, Pseudodiamesa branickii.}

* La zone 3, avec la seule station de Saint-MartinLys $(350 \mathrm{~m})$, représentative des $20 \mathrm{~km}$ à forte pente et à fort courant du Défilé de Pierre-Lys, est assimilable à un métarhithron dont la température maxima atteint $17^{\circ} \mathrm{C}$. Elle est encore caractérisée par 7 Orthocladiinae plutôt rhéophiles :

Cardiocladius capucinus, Eukiefferiella similis, Orthocladius (Euorth.) ashei, O. (Euorth.) rivicola, O. (s. str.) rubicundus, $O$. (s. str.) vaillanti, Paracricotopus niger.

* La zone 4 regroupe les 7 stations aval de l'Aude (St. 6 - 12) réparties sur $80 \mathrm{~km}$ entre Couiza $(220 \mathrm{~m})$ et Trèbes $(84 \mathrm{~m})$ et passant progressivement d'une rivière de piémont d'ordre 4 à une rivière de plaine d'ordre 5 après Carcassonne (St. 10).

La faible pente moyenne $(0,2 \%)$ ralentit le courant lorsque le lit s'élargit au niveau de la station la plus aval de Trèbes (St. 12). Les températures maxima de cette zone atteignent $21^{\circ}$ à $25^{\circ} \mathrm{C}$; elles correspondent à celles d'une rivière de plaine à été chaud.

8 espèces -5 Orthocladiinae +3 Tanytarsini - sont caractéristiques ou préférentes de ce niveau typologique :

Cardiocladius fuscus, *Cricotopus vierriensis, $\mathrm{Na}$ nocladius bicolor, Orthocladius (s. str.) oblidens, *Rheocricotopus chalybeatus, Paratanytarsus dissimilis, *Rheotanytarsus rhenanus, *Tanytarsus brundini.

4 espèces $\left(^{*}\right)$ sont des espèces de plaine $(<600 \mathrm{~m})$ qui colonisent les parties basses (Hyporhithral - Epipotamal) de deux systèmes hydrographiques des Pyré- 
nées centrales et occidentales (Laville \& Vinçon 1991). Il s'agit, pour la plupart, d'espèces modérément eurythermes ou thermophiles.

\section{Discussion et conclusion}

L'analyse statistique de la composition taxonomique et de l'abondance relative des Chironomidés de la haute et moyenne rivière Aude dans les Pyrénées orientales a permis de répartir les 52 espèces les plus fréquentes selon 5 groupes : euryzonales, de source, euryzonales de montagne, de piémont, de plaine. Une station donnée peut abriter une communauté composée d'espèces euryzonales (s. 1.) avec des espèces plus sténotopes et caractéristiques d'une zone particulière. Cette répartition altitudinale a permis de définir 4 zones typologiques successives davantage à l'aide d'espèces préférentes que réellement typiques d'une seule zone.

En fait, la répartition des Chironomidés dans l'Aude apparait plus clinale ou graduelle que zonale ou discontinue. Cette répartition plutôt clinale et la zonation mise en évidence en fonction de la diversité et de l'abondance relative des peuplements confirment le point de vue de Lotspeich (1980) repris par Culp \&
Davies (1982). Dans le cadre d'une analyse de la zonation longitudinale des rivières, ces auteurs suggèrent de combiner le «concept du continuum fluvial » de Vannote et al. (1980) avec un système de classification hiérarchique qui tienne compte du climat, de la géologie, du type de sol et de la végétation du bassin versant.

De même, la zonation longitudinale des Chironomidés de l'Aude paraît tributaire du climat, de la végétation des différents étages - subalpin, montagnard, collinéen, méditerranéen - et de la géologie - axe cristallin siliceux de la chaine, avant pays calcaire - (Fig. 4).

Ainsi, le crénon coïncide avec l'étage montagnard, le métarhithron avec l'étage collinéen calcaire et l'hyporhithron avec l'étage méditerranéen mollassique.

L'importance de la géologie a été montrée par Brauckmann (1987) dans une étude typologique des cours d'eau d'Allemagne où les différences nettes apparaissent dans la structure des biocénoses macrobenthiques en général et des Chironomidés en particulier dans des massifs calcaire et siliceux, pour un même niveau altitudinal depuis la haute montagne jusqu'en plaine.

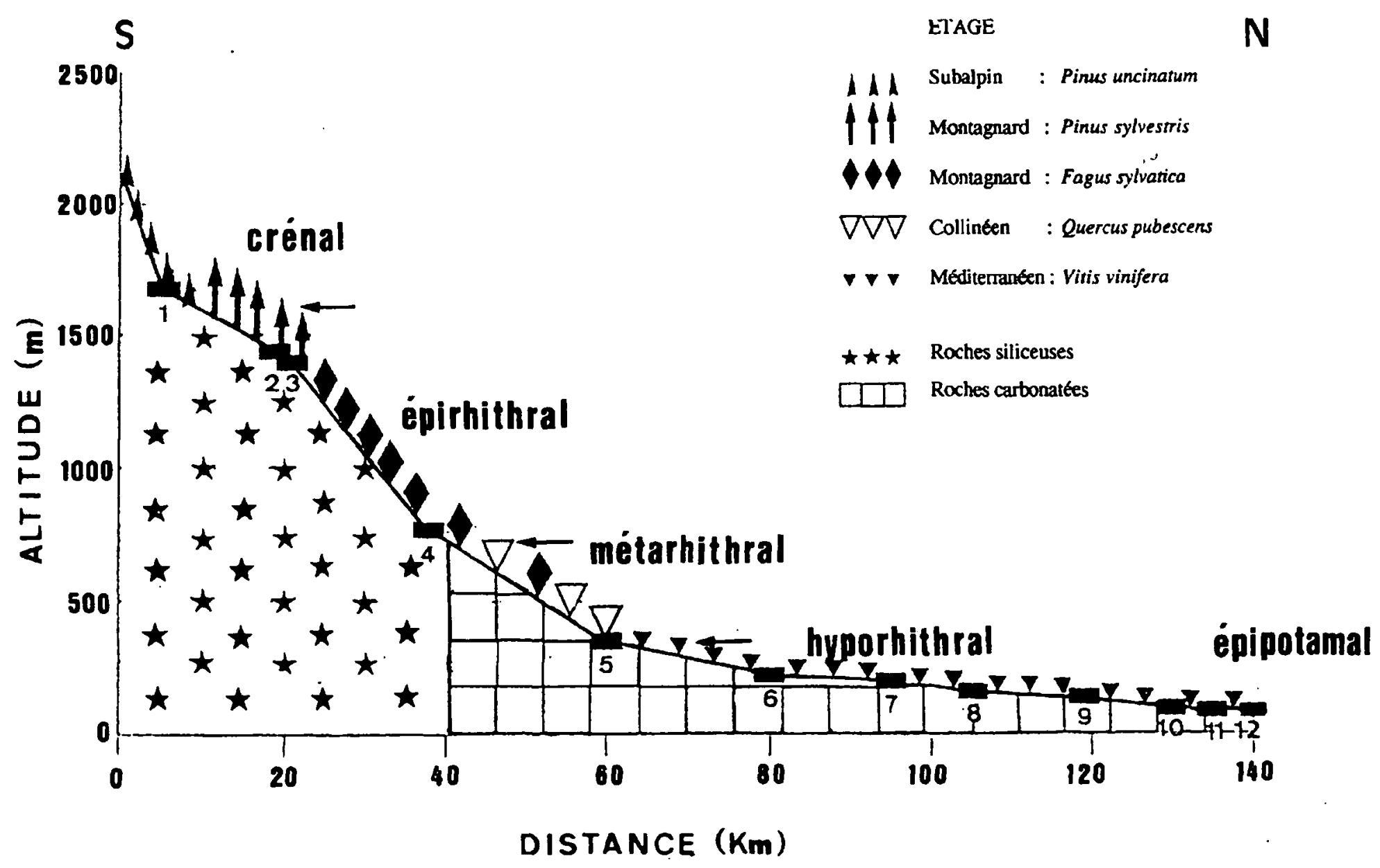

Fig. 4. Profil longitudinal, géologie et étages de la végétation des 4 niveaux typologiques de l'Aude supérieure et moyenne. Fig. 4. Longitudinal section, geology, and vegetation levels of the four typological zones of the River Aude. 


\section{L'Aude : rivière méditerranéenne?}

Parmi les 52 espèces les plus fréquentes de l'Aude, 29 sont euryzonales de même que dans les deux vallées d'Aure et d'Ossau où elles remontent parfois à des altitudes plus élevées $(\rightarrow 2200 \mathrm{~m}$, Laville \& Vinçon 1991).

Par contre, 13 espèces fréquentes (f) dont 9 signalées de piémont $(400-1000 \mathrm{~m})$ et 4 signalées de plaine $(<$ $600 \mathrm{~m}$ ) dans ces deux réseaux pyrénéens colonisent des niveaux plus élevés dans les Pyrénées orientales où elles ont été recensées dans une ou plusieurs des 3 stations supérieures de l'Aude (1400-1700 m). 4 espèces plus rares (r) ont également été récoltées à des altitudes supérieures dans l'Aude (Tableau 7).

Parmi ces espèces, $8\left(^{*}\right)$ n'étaient jusqu'ici recensées que des basses régions avoisinantes des Pyrénées (Zone 13 Limnofauna Europaea) (Laville \& Vinçon 1986). Ces nouvelles récoltes en altitude enrichissent la liste des espèces réellement dans la zone 2 des Pyrénées (>1000 m).

La remontée de ces espèces dans la rivière Aude, de l'ordre de 450 à $1000 \mathrm{~m}$, s'explique en partie par les conditions thermiques estivales plus élevées $\left(25^{\circ} \mathrm{C}\right)$ que dans les Pyrénées centrales $\left(20^{\circ} \mathrm{C}\right)$ pour des zones altitudinales comparables.
Ce réchauffement des eaux pendant l'été est typique des rivières méditerranéennes et influence la répartition altitudinale des invertébrés benthiques. Cette influence climatique sur la remontée d'autres groupes d'invertébrés - Ephéméroptères, Plécoptères, Trichoptères - a été signalée dans plusieurs rivières du pourtour méditerranéen, notamment en Corse, au Maroc et au Liban (Giudicelli et al. 1985). A ce propos, ces auteurs écrivent : " Des taxons thermophiles ou eurythermes, vivant habituellement dans les cours d'eau de plaine ou de piémont s'élèvent.en altitude et occupent une zone altitudinale plus large que dans les réseaux de l'Europe non méditerranéenne».

La situation altitudinale supérieure de 17 espèces de Chironomidés dans l'Aude, comparée à celle des rivières des Pyrénées centrales et atlantiques de même latitude souligne cette influence des conditions climatiques sur la structure de la communauté et le caractère méditerranéen de la rivière.

Ce caractère méditerranéen de l'Aude, également déterminé par des crues violentes pouvant survenir en fin d'été, est cependant atténué par le relèvement de l'étiage estival dû aux lâchers de barrages: et aux sources karstiques de la haute vallée qui atténuent les intermittences des débits. De ce fait, l'Aude ne peut être considérée comme une rivière méditerranéenne typique.

Tableau 7. Remontée altitudinale dans la rivière Aude d'espèces de Chironomidés vivant à des altitudes inférieures dans les vallées d'Aure et d'Ossau. $(f)$ : espèces fréquentes; $(r)$ : espèces rares : $\left(^{*}\right)$ espèces signalées en bordure $(<1000 \mathrm{~m})$ de la zone 2 des Pyrénées (Laville \& Vinçon 1986).

Table 7. Ascent of species in the River Aude normally living in lower zones in the Aure and Ossau valleys of the central and atlantic Pyrenees : frequent $(\mathrm{f})$ and rare $(\mathrm{r})$ species : $\left(^{*}\right)$ species previously signalized in the border $(<1000 \mathrm{~m})$ of the zone 2 of the Pyrenees (Laville \& Vinçon 1986).

\begin{tabular}{lclc}
\hline $\begin{array}{c}\text { Vallées d'Aure et d'Ossan } \\
\text { Espèces de piémont (400-1000m) }\end{array}$ & $\begin{array}{c}\text { Aude } \\
\text { Alt. (m) }\end{array}$ & $\begin{array}{c}\text { Vallées d'Aure et d'Ossau } \\
\text { Espèces de plaine }(<\mathbf{6 0 0 m})\end{array}$ & $\begin{array}{c}\text { Aude } \\
\text { Alt. (m) }\end{array}$ \\
\hline Conchapelopia melanops & $1440(\mathrm{r})$ & * Odontomesa fulva & $1440(\mathrm{r})$ \\
Brillia modesta & $1700(\mathrm{r})$ & Cricotopus bicinctus & $1700(\mathrm{f})$ \\
Cricotopus curtus & $1700(\mathrm{f})$ & $*$ Eukiefferiella ilkleyensis & $1440(\mathrm{f})$ \\
Cricotopus similis & $1700(\mathrm{f})$ & $*$ Rheocricotopus chalybeatus & $1440(\mathrm{f})$ \\
* Eukiefferiella lobifera & $1700(\mathrm{f})$ & $*$ Tanytarsus ejuncidus & $1700(\mathrm{f})$ \\
Eukiefferiella similis & $1700(\mathrm{f})$ & & \\
* Nanocladius rectinervis & $1440(\mathrm{f})$ & & \\
Orthocladius ashei & $1440(\mathrm{f})$ & & \\
Neozavelia fuldensis & $1400(\mathrm{f})$ & & \\
* Rheotanytarsus pentapoda & $1440(\mathrm{f})$ & & \\
* Stempellinella brevis & $1440(\mathrm{f})$ & & \\
Polypedilum laetum & $1440(\mathrm{r})$ & & \\
\hline
\end{tabular}




\section{Remerciements}

Nous remercions N. Giani pour la mise en forme des dendogrammes (Figs. 2 et 3 ) et Mme D. Galy qui a saisi le manuscrit

\section{Travaux cités}

Braukmann V. 1987. — Zoozönologische und saprobiologische Beiträge zu einer allgemeinen regionalen Bachtypologie. Arch. $H y$ drobiol. Ergebn. Limnol., $26: 1-355$.

Culp J.M. \& Davies W.D. 1982. - Analysis of longitudinal zonation and the River Continuum Concept in the Olsman-South Saskatchevan river system. Can. J. Fish. Aquat. Sci., 39 : 1258-1266.

Fittkau E.J. \& Reiss F. 1978. - Chironomidae. In Illies J. Ed. Limnofauna Europaea, 2nd edit. G. Fisher Verlag Stuttgart : 404-440.

Gazagnes G. \& Laville H. 1985. — Etude faunistique des Chironomidés (Diptera) de la Haute Neste d'Aure (Pyrénées centrales) : impact des aménagements hydroélectriques. Annls. Limnol. 21 (2) : 149-159.

Gendron J.M. 1996. - Les Chironomidés (Diptera) de l'Aude, rivière méditerranéenne des Pyrénées orientales. Impact d'une crue catastrophique. Thèse d'Université, U.P.S Toulouse III : 196 p.

Gendron J.M. \& Laville H. 1993. - Diel emergence patterns of drifting chironomid (Diptera) pupal exuviae in the Aude River (Eastern Pyrenees, France). Netherlands journal of aquatic ecology 26 (2-4) : 273-279.

Giudicelli J., Dakki M. \& Dia A. 1985. - Caractéristiques abiotiques et hydrobiologiques des eaux courantes méditerranéennes. Verh. Internat. Verein. Limnol. 22 : 2094-2101.

Langton P.H. 1991. - A key to pupal exuviae of West Palaearctic Chironomidae. Privately published, Huntington, England : $386 \mathrm{p}$.

Langton P.H. 1995. - A key to pupal exuviae of West Palaearctic Chironomidae. Update on the genus Chironomus (1995). Privately published, Huntington, England : 14 p.
Laville H. 1971. - Recherches écologiques sur les Chironomides (Diptera) lacustres du Massif du Néouvielle (Hautes-Pyrénées). Première partie : Systématique, Ecologie, Phénologie. Annls. Limnol., 7(2) : 173-332.

Laville H. \& Lavandier P. 1977. — Les Chironomidés (Diptera) d'un torrent pyrénéen de haute montagne, l'Estaragne. Annls. Limnol., 13(1) : 57-81.

Laville H. \& Serra-tosio B. 1996. - Additions et corrections à l'inventaire des Chironomidés (Diptera) de France depuis 1990. Annls. Limnol. 32(2) : 115-121.

Laville H. \& Vinçon G. 1986. — Inventaire des Chironomidés (Diptera) connus des Pyrénées. Annls. Limnol. 22(3) : 239-251.

Laville H. \& Vinçon G. 1991. - A typological study of Pyrenean streams : Comparative analysis of the Chironomidae (Diptera) communities in the Ossau et Aure Valleys. Verh. Internat. Verein. Limnol. 24 : 1775-1784.

Lebart L., Morineau A. \& Fenelon J.P. 1982. — Traitement des données statistiques, méthodes et programme. $2^{\mathrm{ème}}$ ed. Dunod, Paris : $510 \mathrm{p}$.

Lotspeich F.B. 1980. - Watersheds as the basic ecosystem : this conceptual framework provides a basic for a natural classification system. Wat. resources bull., 16(4) : 581-586.

Serra-Tosio B. \& Laville H. 1991. - Liste annotée des Diptères Chironomidés de France continentale et de Corse. Annls. Limnol., 27(1) : 37-74.

Vannote L., Minshall K.W., Cummins J.R., Sedell J.R. \& Cushing C.E. 1980. - The River Continuum Concept. Can. J. Fish. aquat. Sci., $37: 30-137$.

Ward J.V. 1986. - Altitudinal zonation in a Rocky Mountain stream. Arch. Hydrobiol. Suppl. 74 : 133-199. 